\title{
Calvin et d'Aubigné: vocation prophétique et vocation poétique
}

\author{
DANIEL MÉNAGER
}

Il est toujou rs difficile de parler de poésie religieuse, et encore plus difficile de parler de poésie protestante. Si un poète est conscient de sa mission et de la dignité de la poésie, il acceptera difficilement les directives du magistère, il lui arrivera même souvent de prendre des libertés vis-à-vis de la doctrine. C'est une vérité d'évidence. Et quand il s'agit de poésie protestante, les difficultés redoublent s'il est vrai que Calvin, par exemple, n'était guère enclin à confondre et même à rapprocher prophétie et poésie. Il est normal qu'on s'intéresse à la pensée du Genevois en matière d'art et de littérature, ${ }^{1}$ mais dans le même temps on ne peut oublier que la poésie protestante française s'est développée après lui, et parfois contre lui. ${ }^{2}$ Avec Agrippa d'Aubigné, enfin, personnalité exceptionnelle, poète hors du commun, les formules éclatent: poète biblique, poète calviniste, poète protestant? C'est pourtant les Tragiques que concerneront les pages suivantes. Quelques grandes études en effet, venues de pays différents, en ont renouvelé la compréhension: celle de Richard Regosin sur la poésie de l'inspiration; ${ }^{3}$ en France, celles de Marguerite Soulié ${ }^{4}$ et de Marie-Madeleine Fragonard. ${ }^{5}$ Et l'on n'oublie pas les contributions importantes d'E. Forsyth. ${ }^{6}$ Grands travaux, mais qui présentent des images assez différentes du poète des Tragiques. M. Soulié rattache étroitement la pensée et le style du "bouc du désert" à la foi de Calvin. La thèse de M.-M. Fragonard, en revanche, montre tout ce qu'il doit à la lecture de certains Pères de l'Église et à des courants philosophiques et religieux qui constituent parfois un calvinisme hétérodoxe.

Le propos qui suit se veut modeste. Nous ne chercherons pas une compréhension d'ensemble des Tragiques mais tenterons de confronter les "récits de vocation" de d'Aubigné avec leur source biblique et les commentaires de Calvin: moyen peut-être de mieux comprendre la façon dont d'Aubigné envisage sa mission, ce qu'il demande à Dieu pour la mener à bien, et, finalement, le sens d'une écriture dont on n'a jamais fini de mesurer la complexité. 
Pas de prophétie sans vocation, et même sans "récit de vocation", pour reprendre une expression de Von Rad, auquel sont empruntées les lignes qui suivent: "L'événement de la vocation prophétique a marqué la naissance d'un nouveau genre littéraire, celui des récits de vocation", ${ }^{7}$ possédant malgré leur diversité un certain nombre de thèmes récurrents, et surtout ce que le savant exégète allemand appelle le "style en je": un "je" qui n'est plus le "je collectif inclusif" des anciennes formes cultuelles, mais un "je" expressément exclusif. Ce qui est arrivé au prophète n'est arrivé à personne d'autre, il "rend compte d'un événement qui l'a revêtu d'une mission, d'un savoir et d'une responsabilité, et qui l'a livré à ses seules ressources devant Dieu."

Les Commentaires de Calvin sur les livres des prophètes soulignent l'importance de cette vocation, l'un des signes au reste permettant de distinguer vrais et faux prophètes, ce qui est une obsession de Calvin. On peut mentionner, parmi tant d'autres passages, sa réflexion sur Jérémie 14:14$15 .{ }^{8} \mathrm{Si}$ la vocation, c'est-à-dire l'appel explicite de Dieu prenant au reste des formes différentes selon les cas, s'avère nécessaire, c'est bien parce que la prophétie comble une défaillance, celle du sacerdoce. ${ }^{9}$ Les prophètes se lèvent quand les prêtres abdiquent et oublient les devoirs de leur tâche. Et d'une certaine façon, ce que Calvin écrit des prophètes de l'Ancien Testament se vérifie aussi dans son cas, lui qui fut presque malgré lui prophète de l'Évangile du Christ. ${ }^{10}$ Peu porté à parler de lui-même, Calvin nous a laissé, dans sa Préface aux Commentaires sur les Psaumes un véritable récit de sa vocation, fidèle pour l'essentiel aux lois du genre. Pas de révélation, sans doute, ni de parole de Dieu directement adressée à lui, mais la représentation d'un événement, la réalité d'un appel et un bouleversement total de sa vie. ${ }^{11}$

Ce récit de vocation se trouve aussi dans les Tragiques, et même à plusieurs reprises, notamment dans les prologues des chants I, II et VI. L'appel vient de Dieu:

Mais le doigt du grand Dieu me pousse à le combattre ${ }^{12}$

ou de son Église, la "captive Eglise",

Qui à sa delivrance (aux despens des hazards)

M’appelle, m'animant de ses trenchans regards. ${ }^{13}$

La thématique des récits de vocation bibliques apparait souvent, la peur par exemple devant l'immensité de la tâche voulue par Dieu et les ennemis qui guettent le prophète, cette peur qu'incarne bien sûr Jonas au début du chant VI et que seule peut vaincre la confiance en Dieu; la peur encore, évoquée dans un passage des Fers sur Élie réfugié auprès du torrent de Carith 
pour fuir la colère d'Achab. ${ }^{14}$ Et comme les auteurs bibliques, comme Calvin lui-même, d'Aubigné sait découvrir dans le passé et dans le présent $t^{15}$ les merveilles de la Providence de Dieu pour ceux qui le craignent. Sur deux points cependant la mise en scène de la vocation s'écarte de la "vulgate" biblique. Si le prophète est faible, l'aide de Dieu le rend redoutable, et d'Aubigné insiste davantage sur cet aspect de l'activité prophétique que sur la déréliction, prenant plaisir, par exemple, à rappeler les "vengeances" exercées par

\section{les foibles puissances}

Des femmes, des enfans, des vallets desreglés.

Des Gedeons choisis, des Samsons aveuglés. ${ }^{16}$

Thème prophétique et en outre paulinien ("ma puissance donne toute sa mesure dans la faiblesse", II Cor. 12:9), mais dont l'ambiguitté ici est réelle. Met-il l'accent sur la puissance de Dieu, ou sur celle du prophète revêtu de sa force? Le volontarisme et la fureur de certains vers (le prologue de Princes en particulier) paraissent bien éloignés de la frayeur sacrée et du sentiment d'impuissance qui étreint souvent les prophètes (Jérémie par exemple) et que Calvin n'a pas manqué de souligner dans ses Commentaires. ${ }^{17}$ Cette perspective trouve peut-être son explication dans le fait que le poète des Tragiques ne vit pas la même rupture que les prophètes bibliques ou même ceux des temps modernes. "Ce qui est particulièrement caractéristique, écrit encore Von Rad, c'est l'absence totale de transition d'un état à l'autre. Être prophète n'était pas le résultat d'une escalade prodigieuse ni d'un dépassement de l'existence religieuse antérieure. Devant Dieu ce n'est pas la foi dont il a déjà fait preuve, ni aucune autre aptitude qui qualifient l'appelé pour sa nouvelle vocation." 18 Or c'est bien une aptitude qui qualifie d'Aubigné pour affronter les "légions de Rome": ses dons poétiques. ${ }^{19}$ L'opposition établie par l'auteur entre la poésie profane qu'il écrivait avant et celle qu'il écrit maintenant, après avoir entendu l'appel de Dieu, ${ }^{20}$ ne fait que souligner une continuité: toujours la poésie. Ailleurs, en des vers bien connus, d'Aubigné se montre devenu "plus sage":21 image du progrès moral. Et le prologue de Princes fait appel pour sa part à l'image d'une croissance: la voix donnée par Dieu trouve maintenant sa véritable destination, de louange et de fureur. Mais elle a été donnée dès la naissance comme à tous les poètes qui "naissent". Voici le poète pourvu sans doute d'un "nouveau cœur", 22 mais il reste lui-même grâce à la poésie qui fait partie de sa nature.

Le prologue de Vengeances conduit peut-être à nuancer le propos: son accent différent, ses thèmes aussi, amènent $\mathbf{M}$. Soulié à discerner deux attitudes prophétiques successives correspondant respectivement aux livres I à Vet aux livres VI à VII "Après l'éclat des trompettes, voici l'harmonie 


\section{8 / Renaissance and Reformation}

pénétrante de la prière", et de relever dans les premiers vers du chant VI "un mouvement de repentance (...) une véritable "metanoia", une conversion totale du coeur et de la pensée. Le prophète des premiers chants, nouvel Esaïe, nouveau Moyse qui a lancé l'anathème contre les ennemis du Seigneur (...) devient maintenant l'humble servant de l'Evangile."23 L'homme qui demande à Dieu de le rendre mort au monde, de lui donner la grâce d'u ne nouvelle naissance (selon la parole du Christ à Nicodème) ${ }^{24}$ n'a plus grand chose à voir, en effet, avec le prophète casqué du livre I. Différence explicable, toujours selon M. Soulié, par les dates auxquelles ces deux prologues ont été écrits et que sépare une longue évolution spirituelle depuis l'espoir d'une conquête protestante jusqu'à l'immense épreuve constituée par les signes avant-coureurs de l'abjuration d'Henri IV, ce qui conduit l'auteur à dater le prologue de Vengeances de 1586 environ. ${ }^{25}$

En tout état de cause, on ne peut dissocier la question de la vocation prophétique chez d'Aubigné des moyens qu'elle se donne: le songe et la vision. Au début du chant VI, paraphrasant le prophète Joël (II, 28-29), d'Aubigné s'écrie à l'adresse du Seigneur:

Comme tu as promis, donne en ces derniers ans Songes à nos vieillards, vision aux enfans. ${ }^{26}$

Et chacun sait par ailleurs que les Tragiques sont issus d'une vision, celle de Talcy, racontée à la fin des Fers. Il s'agit là d'un choix, sur lequel on peut s'interroger.

"Songes" et "visions": la littérature philosophique et théologique de l'époque s'est employée avec plus ou moins de bonheur à distinguer les deux termes. ${ }^{27}$ En principe, le songe est une espèce d'un genre plus général: la vision. Il ne se produit que pendant le sommeil, alors que la vision peut intervenir à tout moment, sommeil ou veille, nuit ou jour. ${ }^{28}$ Bodin essaie comme beaucoup d'établir une hiérarchie entre les différentes formes de révélation, et il s'appuie lui aussi sur le passage de Joël pour proposer une relation entre jeunesse et vision, vieillesse et songe: "C'est pourquoy on list en Joël le Prophète, que aux derniers jours les jeunes auront des visions, et les vieux auront des songes. Or le songe est beaucoup moindre que la vision." ${ }^{29}$ D’Aubigné, quant à lui, au moins dans ce prologue de Vengeances, tout en abandonnant l'idée de hiérarchie ${ }^{30}$ entre les différentes formes de révélation, fait jouer les idées d'âge et de pureté. Vieillards et enfants symbolisent le dénuement et la pauvreté.

Il importe toutefois de replacer ce passage dans la tradition protestante. Luther et Calvin ont commenté l'un et l'autre les versets de Joël et dans un sens qui n'est pas celui de d'Aubigné. Pour Luther, Joël prophétise la 
Pentecôte où l'Esprit sera donné à tous: "Le don du sainct Esprit est donné indifferemment à tous ceux qui invoquent le nom du Seigneur, soyent femmes ou hommes, vieils ou jeunes, serfs ou francs." ${ }^{11}$ On ne peut être plus clair. Calvin ne l'est pas moins, insistant avant tout sur l'effusion de l'esprit: "Significat igitur vulgo omnes fore participes gratiae Spiritus." ${ }^{2} \mathrm{Et}$ encore: "Nunc autem propheta ostendit donum illud et gratiam ad omnes ordines." ${ }^{33}$ On ne peut imaginer divergence plus grande. Pour Calvin, Dieu accorde ses révélations à tous ceux qui se nourrissent de sa parole, et l'âge ne possède aucun privilège, tandis que le poète des Tragiques attribue aux deux extrêmes de la vie des atouts métaphysiques qui sont de véritables privilèges. ${ }^{34}$ Sa perspective apparaît plus philosophique que théologique. On ne peut la comprendre sans recourir à un dualisme qui fait du corps la prison de l'âme, empêchée par lui de voler jusqu'aux cieux (vers 28 ), ${ }^{35}$ et qui explique pourquoi l'enfance et la vieillesse, âges où le corps est faible, se trouvent valorisées. À quoi sans doute on objectera que l'enfance est dans ces vers un état d'esprit, une grâce autant qu'un âge, la chance d'une nouvelle naissance selon la parole du Christ à Nicodème. Même si cette interprétation est correcte, elle rapproche la pensée de d'Aubigné de celle des Libertins spirituels qui avaient sans cesse à la bouche le vocabulaire de la régénération et le thème, justement, de l'enfance ${ }^{36}$ - qu'on retrouve d'ailleurs dans le Théâtre de celle qui passa pour leur protectrice: Marguerite de Navarre. ${ }^{37}$

Luther d'autre part ne s'attardait guère sur les différences entre songe et vision. "C'est tout un de prophétie, vision ou de songes, c'est-à-dire de cognoissance de Dieu par Christ, laquelle le Saint Esprit fait reluire par la parole de l'Evangile." 38 Pas de révélation en dehors de la Bible, illuminée par l'Esprit, et le Réformateur allemand s'en prend très clairement aux anabaptistes qui attendent de nouvelles révélations. ${ }^{39} \mathrm{La}$ vigilance de Calvin n'est pas moindre comme le montre fort bien son commentaire de Daniel 7, qui insiste moins sur le privilège constitué par la vision que sur sa difficulté; il présente un prophète terrassé par la manifestation du Seigneur et qui déchiffre mal ce qu'il voit: "Simul etiam docemur exemplo prophetae, non esse cur repudiemus omnes visiones, sed petamus a deo ipso interpretationem." 40 Calvin se sert au fond de ce passage pour faire comprendre que l'intelligence des choses divines et en particulier celles de l'Écriture excèdent notre entendement, et qu'il faut en demander le sens "ab Christo, qui familiariter hodie nos docet par pastores et ministros evangelii." 41 Quant à l'attente ou l'espoir d'une vision, Calvin reste très ferme: "Hodie Deus non loquitur nobiscum per visiones sed vult nos contentos esse lege sua et evangelio." ${ }^{2}$ Sa prudence va si loin qu'il n'admet même pas les visions consolatrices, celles par exemple que Dieu peut offrir aux martyrs. Ceux du temps présent ne doivent pas espérer voir, comme Étienne (Actes 7:55), le ciel ouvert au-dessus d'eux. Dieu les console autrement: 


\section{0 / Renaissance and Reformation}

"Non quod per externam visionem nobis appareat, sicuti Stephano, sed ita se intus nobis patefaciet, ut ejus praesentiam vere sentiamus. Atque hic modus videndi nobis sufficere debet, quum Deus sua virtute et gratia non modo se propinquum esse demonstrat, sed etiam probat se in nobis habitare." 43 Voilà le véritable mysticisme de Calvin, présenté parfois, et à tort, comme étranger à toute forme de sensibilité religieuse.

Il faut croire cependant que l'admirable rigueur de Calvin n'a pas suffi à d'Aubigné. Avec lui s'opère un passage rapide du prophétique à l'apocalyptique. Passons sur le fait que le poète octroie à certains de ses martyrs la vision d'Étienne: c'est le cas par exemple d'Anne Askève qui s'écrie dans les Fers:

Voici les cieux ouverts, voici son beau visage. ${ }^{44}$

Le récit de son supplice dans le Martyrologe de Crespin s'en gardait bien..$^{45}$ Mais c'est la vision même d'où est sorti le poème qui s'écarte dangereusement de la tradition calvinienne. D'Aubigné adopte une position courante dans la littérature de type apocalyptique, qu'elle vienne de la Bible ellemême ou d'autres traditions. Beaucoup plus que chez les prophètes, qui sont plutôt les hommes de la chose entendue, la vision est, dans le genre apocalyptique, le point de départ du discours. ${ }^{46} \mathrm{Et}$ surtout, le ravissement du poète lui donne accès aux secrets de l'histoire ${ }^{47}$ constitue une échappée vers la fin de celle-ci. Il n'est pas étonnant que les vers qui le décrivent soient empruntés à Daniel. Mais si "le prophète domteur des lions indomptés" représente bien l'Apocalypse de l'Ancien Testament, il y a loin entre son attitude et celle du poète quand le futur leur est dévoilé. Ils ont sans doute recours l'un et l'autre à un intercesseur, ${ }^{48}$ et c'est l'Ange qui explique les tableaux célestes. Mais Calvin insistait sur l'anéantissement du prophète biblique dont il tirait une leçon toute spirituelle: "Nous sommes admonnestés par cet exemple là, que pour bien comprendre les secrets de Dieu qu'il faut que nous soions comme abatus et aneantis en nous." 49 D'Aubigné évoque quelque chose de très différent: une extase ("En cependant qu'en luy, exstatic, je me pasme.", v. 1205), ${ }^{50}$ c'est-à-dire une profonde jubilation de l'âme.

L'objection essentielle de la Réforme à l'apocalyptique est qu'elle succombait au péché de curiosité. Péché par excellence pour Calvin, parce qu'elle dépouille Dieu de sa transcendance. C'est contre elle qu'il met en garde quand il commente les révélations de Paul (II Cor. 12), ${ }^{51}$ et c'est elle qui lui paraît l'erreur fondamentale de l'apocalyptique. Il n'est peut-être pas exact de dire par conséquent que les Réformés du XVIe siècle "ne semblent pas avoir établi de distinction de genre entre prophétie et apocalyptique." 52 Rappelons à ce sujet que Luther s'est résigné difficilement à admettre l'Apocalypse de Jean dans le canon des Écritures; ${ }^{53}$ que c'est un 
des très rares livres de la Bible que Calvin n'ait jamais commentés. Pourquoi d'ailleurs faudrait-il oublier ce que Bullinger écrit dans la préface à ses Sermons sur l'Apocalypse et qui constitue une référence très précise aux réticences de beaucoup à l'égard des "visions, pourtraits et figures" 54 inséparables du genre? L'Avertissement au lecteur qui précède l'Apocalypse de Jean dans une édition genevoise est encore plus précis, car c'est une mise en garde contre les spéculations en tout genre alimentées parl'Apocalypse. ${ }^{55}$ On retrouve ici l'esprit de Calvin dans son propre commentaire au livre de Daniel, ainsi résumé par Cl. G. Dubois: "Il ne s'agit pas (dans le livre de Daniel) d'une (...) anticipation historique (...) Quel en est le sujet? Il n'est pas historique, il est moral. Les révélations historiques sont au service d'une volonté divine de consolation (... ) Dieu ne révèle de l'histoire que ce qui est conforme à cette intention." Et encore ceci: "Il y a donc chez Calvin une volonté de lutter contre le courant apocalyptique de son époque." 56

Une partie de la Réforme française n'a pas écouté ces avertissements. Le développement des luttes explique pourquoi, et de plus en plus, elle sollicite l'Apocalypse: pour savoir "jusques à quand?" Dieu éprouvera son peuple. C'était retrouver la fonction parénétique du genre. Chez d'Aubigné le recours à l'apocalyptique s'explique aussi par là. Il suppose aussi une volonté de connaissance inséparable de sa conception de la poésie.

Le "je" du prophète, selon Von Rad, est un "je" exclusif. Celui de d'Aubigné aussi, mais pour des raisons différentes. Ce qui le met à part, ce n'est pas l'ordre de Dieu et l'incompréhension des hommes devant sa mission, mais c'est une entreprise qui n'eut jamais d'exemple. Elle trouve sa figure dans le "chemin tout neuf" du début de Misères (v. 19), chemin où il s'aventure seul, guidé sans doute par les astres de Dieu, mais dans une situation bien différente de celle de la Bible. Moïse conduit un peuple: qui peut suivre le poète dans son aventure solitaire? Si les références bibliques de ce passage ont été bien relevées et commentées, on a omis, semble-t-il, de signaler une réminiscence plus inattendue, celle de Lucrèce, engagé lui aussi dans une poésie nouvelle (I, $921 \mathrm{sqq})$ et qui usait déjà de l'image du chemin non frayé. Ce n'est pas seulement la mission religieuse que ces vers mettent en valeur, c'est l'originalité et l'invention littéraires, comme le montre aussi le "bien plus haut" 57 de ce prologue.

Il serait trop facile d'opposer ici le souci de la poésie et celui de la vérité divine. Celle-ci peut ennoblir celle-là sans qu'il y ait la moindre contradiction. Remarquons cependant un vocabulaire qui est celui de l'audace et qui rappelle peut-être que nous devons le texte des Tragiques au "larron Prométhée", figure de l'éditeur. La disposition même du premier prologue 
place dans un ordre intéressant la décision et l'appel. Au mépris d'une certaine logique, c'est la décision qui est dite en premier, l'image de la captive Église n'intervenant qu'après les douze premiers vers pour appeler le poète à son secours. La mise en scène de la mission laisse enfin au poète une totale liberté - et c'est là l'essentiel. La "captive Eglise" se contente de tourner vers lui ses "trenchans regards", ${ }^{58}$ elle ne dit rien, alors qu'il eût été possible d'imaginer, comme dans une épopée, les paroles que le poète aurait eu à redire..$^{59}$ Celui-ci choisit une mise en scène où il n'a rien à répéter. Il n'est donc pas l'envoyé de l'Église, son mandataire.60

La situation se modifie sans doute au chant $\mathrm{V}$ lorsque l'Ange après avoir commenté les tableaux célestes, donne un ordre explicite au poète: "Escritz fidellement" (V, 1422). Et l'Ange, à la différence de l'Église, tient un long discours qui constitue à la fois l'envoi du poète et le référent de l'œuvre qu'il va composer. Pourquoi faut-il alors que d'Aubigné marque une "pose" (v. 1443) et qu'avant de décrire les derniers jugements de Dieu, il introduise à la fin du chant une autre vision, celle du vieillard Océan? Car cette vision là ne provient pas du "céleste voyage", elle ne fait pas partie des tableaux commentés par l'Ange. D'Aubigné la justifie par le "congé" que l'extase a donné à l'esprit,

De ne suivre, escrivant, du vulgaire la phrase. ${ }^{61}$

La vision ainsi présentée devient une figure de la poésie, le vulgaire étant renvoyé à la prose. Et d'Aubigné se sert de la première vision, de type mystique, pour autoriser la seconde, de type poétique. Il se sert de ce qui lui a été donné pour justifier ce qu'il invente. Si la première vision ne peut se soustraire, en principe, aux exigences de la fidélité, la seconde n'a que faire de celle-ci, puisqu'elle est totalement l'oeuvre du poète. La mystique ici dégénère en poétique.

D'Aubigné ne rend finalement de comptes qu'à lui-même. C'est aussice qu'on peut lire dans l'examen de conscience, au sens propre, du début du chant IV. Un songe matinal lui fait voir sa conscience,

Qui au visage avoit les traits de mon visage. ${ }^{62}$

Ce songe, à la différence de la vision du chant $\mathrm{V}$, ne fait pas découvrir un visage tout autre, occasion de souligner, avec M.-M. Fragonard, combien d'Aubigné s'écarte d'une tradition qui, avec saint Augustin, faisait du songe matinal l'ouverture à la lumière divine ${ }^{63} \mathrm{Et}$ c'est devant lui-même, vu en miroir, que le poète plaide la cause de sa poésie, car il doit expliquer comment il ose choisir parmi tous les martyrs de Dieu. D'où un partage des tâches: à l'histoire l'exhaustivité, à la poésie le droit d'être élective. Que peut signifier dans ces conditions la fidélité de l'écriture? "J'escris fidellement", 
maintient d'Aubigné (v. 51). Mais il ne s'agit plus ni de fidélité à un discours antérieur, ni de fidélité au réel. Ce que la poésie affirme, c'est sa liberté, dont elle n'a de comptes à rendre à personne sinon à la "conscience".

Le poète s'octroie enfin une autre liberté dans la relation qu'il établit entre vision et parole. Il est inutile de refaire la belle démonstration de $\mathbf{M}$. Jeanneret sur le sens de cet appel à la vision. ${ }^{64}$ L'image visuelle possède chez d'Aubigné le prestige qui est le sien chez les néo-platoniciens. "L'intelligible des néo-platoniciens est inséparable de sa manifestation concrète." Cette proposition, on le sait, est due en particulier aux analyses si remarquables de E.H. Gombrich. ${ }^{65}$ Et si le visuel l'emporte sur le textuel, s'il est un mode de connaissance proprement divin, c'est parce que dans la vue, la réalité se donne d'un seul coup. Dans les moments de ravissement "le génie reçoit une vision fugitive de l'Idée platonicienne", "vision immédiate", sans les béquilles du "raisonnement discursif.." ${ }^{66}$ D'où le privilège accordé par Ficin à la vision de Paul sur le chemin de Damas, vision identifiée par lui à l'extase platonicienne. Dans ces conditions, écrire la vision c'est retomber dans le temps. On se doute que Calvin ne voit pas du tout les choses ainsi. D'abord parce que Dieu continue pour lui à se révéler surtout "ex auditu"; ensuite parce que, s'il procède autrement, il est nécessaire d'écrire ce qu'on a vu pour le transmettre aux autres, et sans se poser de questions sur l'ineffable. C'est ce qu'a fait Daniel: "His verbis docet se non privatim vidisse somnium sua causa, sed in communem ecclesiae aedificationem. Qui existimant Danielem repente exsiliisse e lecto, ne oblivisceretur somnii, frivolem et inane commentum afferunt." ${ }^{67} \mathrm{Du}$ songe à l'écriture, il n'y a pas déperdition, car Calvin s'intéresse essentiellement au message.

Il n'en va pas de même chez d'Aubigné. Si la vision demeure le modèle du poème, celui-ci lui demeure irréductible - ce qu'a fort bien vu M. Jeanneret: "Le poème (... ) se déroule dans le temps, il accepte les lois de la succession et de la mort (...) Ce que le tableau concentre, le texte le disperse et le dilue." ${ }^{68}$ Cette dispersion serait périodiquement contrôlée par l'apparition de "points de surplomb" qui restaureraient une vision spirituelle. Mais ce qui sans doute est premier dans le travail et la mise en scène de l'écriture, c'est un type d'image qui ne doit rien aux visions célestes. Le prologue du chant I - rappelons-le - met en scène la "non-commune image"69 qui accompagne le poète dans la composition de son œuvre. "Non-commune" sans doute parce que c'est celle de ruisseaux de sang; "noncommune" aussi parce qu'elle correspond très exactement aux "imagines agentes" des traités sur la mémoire et des rhétoriques qui s'y intéressent. ${ }^{70}$ Elle fait partie des " $\varphi$ ov $\tau \alpha$ ơ $\alpha i$ " qui permettent à l'orateur d'être éloquent parce qu'il est passionné. Elle fournit celui-ci en sentiments et en émotions plus qu'en mots. Mais si cette analyse est juste, elle nous introduit à 
une psychologie plus qu'à une théologie de l'écriture. Du même coup, la question de la fidélité se pose en termes différents. Il ne s'agit plus d'être fidèle à la parole d'un ange ou à ce qu'une extase a permis de voir. La représentation fournie par l'imagination double (dans tous les sens du terme) celle qui venait d'en haut. La vision mystique ne serait-elle pas alors la caution transcendante cherchée par le poète en mal de justification? Tel pourrait être son rôle dans ce théâtre de l'écriture.

D'Aubigné n'est sans doute pas le premier poète protestant à s'écarter de Calvin. Voici déjà quelque temps, Mario Richter avait montré, sur des exemples contemporains, que telle était bien la tendance de la poétique réformée dans la seconde moitié du siècle. ${ }^{71} \mathrm{La}$ situation de d'Aubigné n'en reste pas moins exemplaire. Pour tenir le rôle du prophète tel que le voit Calvin, il n'avait pas besoin de cet appel à la vision et au songe. Il lui suffisait de savoir déchiffrer l'histoire à la lumière de l'Écriture. Telle était l'attitude pastorale. Elle ne pouvait convenir à un poète en qui demeure très vive la conception de l'inspiration poétique proposée par la Pléiade et qui, finalement, croit au privilège qu'elle donne au poète, homme de la solitude moins parce que Dieu l'a élu que parce qu'il a lui-même choisi cette voie. Les Tragiques évoquent plus d'une fois un cheminement religieux personnel qui aurait fait froncer les sourcils de Calvin. ${ }^{72} \mathrm{Ce}$ que d'Aubigné escamote finalement, par ses appels à la vision, c'est rien moins que l'Écriture. Tel est l'un des effets de sa passion de l'immédiat. De cette passion de l'immédiat relève aussi son impatience à l'égard de l'histoire, et son anticipation, par l'apocalypse, de la fin des temps. En même temps d'Aubigné fait à la rhétorique et à ses techniques une place de choix. C'est elle qui lui permet de dépasser la nostalgie de la vision et du "repos de Capoue" qui laisse rapidement la place à l'éloquence militante. Origine théologique de l'œuvre, visée de l'âme fatiguée par l'histoire, la vision représente aussi, peut-être même surtout, la garantie mystique nécessaire à une œuvre qui s'interroge toujours - c'est là sa grandeur - sur sa raison d'être.

\section{Université de Paris}

\section{Notes}

1 Le livre fondateur sur ce sujet reste, à notre avis, celui de L. Wencelius, L'Esthétique de Calvin (Paris: Les Belles Lettres, 1937).

2 Voir en particulier M. Jeanneret, Poésie et tradition biblique au XVI siècle (Paris: Corti, 1969).

3 Richard Regosin, The Poetry of Inspiration: A. d'Aubigné's les Tragiques (Chapell Hill: University of North Carolina Press, 1970).

4 Marguerite Soulié, L'Inspiration biblique dans la poésie religieuse d'A. d'Aubigné (Paris: Klincksieck, 1977). 
5 Marie-Madeleine Fragonard, "La Pensée religieuse d'A d'Aubigné et son expression", thèse d'État, dactylographiée, Université de la Sorbonne nouvelle, 1981.

6 En particulier: E. Forsyth, "Le message prophétique d'Agrippa d'Aubigné", B.H.R.XII(1979), pp. 24-39; et E. Forsyth, "D'Aubigné, Calvin et le comble des péchés", Mélanges V. L. Saulnier (Genève: Droz, 1984), pp. 263-272.

7 Gerhard Von Rad, Théologie de l'Ancien Testament (Genève: Labor et Fides, 1965), t. II, p. 49.

8 Calvini Opera, éd. Baum-Cunitz (Brunsvigae, 1863-1900), t. XXXVIII, col. 192.

9 Voir en particulier les commentaires sur Jérémie 14:14-15 (t. XXXVIII, col. 192) et sur Michée 3:12: "Diximus alibi factum fuisse Sacerdotum ignavia, ut Prophetae illis (=les prêtres) adjuncti fuerunt." (XLIII, col. 333).

10 La comparaison entre la situation de l'Église à l'époque de Calvin et certains moments de l'histoire biblique est établie souvent par le Réformateur de Genève: voir encore le commentaire à Michée 3:12: “Oportuit quasi extra ordinem Prophetas excitari." Sur les relations entre le prophétisme et le sacerdoce, voir A Neher, L'Essence du prophétisme (Paris: PUF, 1955).

11 Calvini Opera, t. XXXI, col 22. Voir D. Ménager, "Théodore de Bèze, biographe de Calvin", B.H.R. t. XLV (1982), pp. 231-255.

12 Princes, v. 42.

13 Misères, v. 16-17.

14 Fers, v. 1175-1182; voir I Rois 17:6.

15 Comme dans le cas de Merlin, pasteur attaché à la personne de Coligny et que Dieu assiste comme il le fit pour Élie dans l'épisode cité note 14.

16 Vengeances, v. $441-442$.

17 Et encore plus dans certains sermons, celui-ci par exemple sur les visions de Daniel: "Nous sommes admonnestés par cet exemple là, que pour bien comprendre les secrets de Dieu qu'il faut que nous soions comme abatus, et aneantis en nous, car nous voyons nostre nonchallance, et comme nous sommes paresseux (...). Pour avoir la vraie intelligence des secrets de Dieu ( . . . ) il ne faut pas que nous y venions avec une presomption de nostre esprit ( ... ). Il faut que nous soions pleinement abatus, c'est à dire que tout ce que nous avons de nostre nature defaille." (Sermon XIV, t. 41, col 468).

18 Op. cit., p. 52.

19 D'Aubigné l'indique très clairement quand, dans le prologue de Vengeances (v. 107), il se réfêre à la parabole évangélique des talents (Mt. 25:14-30).

20 Voir en particulier Misères, v. 55 sqq. et Princes, v. 59-84.

21 Misères, v. 55.

22 Princes, v. 38.

23 Op. cit., p. 197.

24 Vengeances, v. 40 et Jean 3:3 et 5.

25 Les arguments de $\mathbf{M}$. Soulié ont été discutés par E. Forsyth, "Le message prophétique d'A. d'Aubigné", pp. 25 sqq.

26 V. 15-16.

27 Voir les analyses très précises de M.-M. Fragonard, op. cit., ch. XI.

28 M.-M. Fragonard, p. 869. La distinction opérée par Macrobe (Commentaires sur le songe de Scipion, I, 3) est d'un type différent. Le propre du songe est de nous faire ses communications "dans un style figuré, et tellement plein d'obscurités qu'il exige le secours de l'interprétation" alors que la vision "a lieu lorsque les personnes ou les choses que nous verrons en réalité plus tard se présentent à nous telles qu'elles seront alors." Voir F. Hallyn, "Le Songe de Kepler", B.H.R. t. X XLII 190, pp. 329-247, et A.H.M. Kessel, "Ancient Systems of Dream-Classification", Mnemosyne (1969), pp. 389-424.

29 Démonomanie, deuxième édition (Paris: J. du Puys, 1581), f 25 vo. 


\section{6 / Renaissance and Reformation}

30 Elle existe cependant si l'on prend l'œuvre dans son ensemble: au plus bas degré, la vision qui passe encore par limagination, comporte des corps et des formes venus du monde sensuel et demande à être décryptée; au-dessus, l'extase où seuls Dieu et les anges peuvent se manifester; enfin la vision qui est fusion avec l'Intellect divin (M.-M. Fragonard, p. 869).

31 Martin Luther, Commentaires sur les revelations des prophetes Joël et Jonas, tr. fr., J. Crespin, (1558), pp. 129-130. Pour Luther, le don du Saint-Esprit est bien supérieur à celui de prophétie qui peut être accordé à des méchants (p. 133).

$32 \mathrm{~J}$. Calvini praelectiones in duodecim prophetas ... minores (Genève: 1559), p. 204.

$33 \mathrm{Ibid} .$, p. 204. Calvin explique que les prophètes, à l'époque de l'Ancien Testament, étaient rares: "Scimus etiamsi olim Prophetae habuerint sua collegia, tamen fuisse paucos numero." (p. 204).

34 Sur le prestige moral et religieux des vieillards, voir A. Thierry, "Les vieillards dans l'Histoire Universelle d'A d'Aubigné", Études seiziémistes (Genève: Droz, 1980), pp. 371-179.

35 Il faut noter, avec M.-M. Fragonard (pp. 68 sqq.), que l'Avis aux lecteurs et la Préface présentent le poète sous la figure de l'Initié.

$36 \mathrm{Ce}$ que Calvin stigmatise dans l'opuscule écrit contre eux (s.1. 1547) objectant que la vraie régénération est la pénitence, et non un état angélique (p. 108).

37 Voir le rôle des enfants dans certaines pièces du Théâtre profane, en particulier dans la comédie de l'Inquisiteur.

38 Commentaires sur les revelations des prophetes Joël et Jonas, p. 134.

39 Ibid., p. 133.

40 Commentaires du livre de Daniel, C.O. XII, col 65.

41 Ibid.

42 Ibid.

43 Commentaires des Actes des Apôtres, C.O., t. XILVIII, col. 167. Et aussi: "Unde sequitur, non in coelis, sed potius in ejus oculis editum fuisse miraculum. Quare non est quod hic multum disputemus de visione physica, quia certum est minime naturali ordine vel modo, sed novo et singulari ratione visum illi fuisse Christum." (col 168 ). Voir le commentaire de M. Soulié,op. cit., p. 142.

44 V.201. Cette vision des cieux ouverts se retrouve dans le grand discours de Montalchine (Feux, v. 812) avec une présentation plus calvinienne (v. 847).

45 Histoire des martyrs, éd. de 1597, fo 172-176 vo.

46 Le prophétisme lui-même connaît cependant une évolution et les visions abondent chez les derniers prophètes, comme d'ailleurs chez Ézéchiel.

47 Dans un article récent ["Le Cinquième sceau. Les tableaux des Fers et la perspective apocalyptique dans les Tragiques d'A d'Aubigné", Mélanges V. L. Saulnier (Genève: Droz, 1984)], A. Tournon distingue à juste titre "deux points de vue possibles sur l'histoire humaine interprétée selon la foi.": l'une privilégiant la Providence et s'attachant aux causes et aux moyens; l'autre, apocalyptique, cherche par le dévoilement qui s'opère à la fin des temps le sens des épreuves. La seconde perspective, qui est celle de Talcy, "interdit que l'espoir ne dégénère en optimisme, la foi en pseudo-savoir. Elle prolonge, dans l'espérance même et dans l'attente de Dieu, la tragédie toujours recommencée des persécutions et des défaites." (p. 280). Cette distinction, tout à fait pertinente et féconde, n'empêche que le visionnaire revient au monde non seulement avec une espérance mais aussi avec un savoir concernant le cours de l'histoire (Fers, 12751416) et porteur de "secrets" (Fers, v. 1200). La pratique de l'apophétie (attestée chez le prophète Daniel lui-même) permet ce savoir.

48 Daniel 7:16.

49 Voir la note 17.

50 La vision des Fers est d'autant plus anticipatrice que ce vers même annonce les deux derniers vers des Tragiques qui concernent, eux, la Parousie: 
Tout meurt, l'ame s'enfuit, et reprenant son lieu Exstatique se pasme au giron de son Dieu.

51 "Si quis roget quaenam (=verba) fuerint, responsio brevis, non abs re vocari ineffabilia, et quae nefas est proferre. Verum excipiet quispiam supervacuum igitur et inutile quod Paulus audierit. Quorsum enim igitur audiebat, quod esset perpetuo silentium premendum? Respondeo, id factum fuisse Pauli ipsius causa. Nam quem manebant tam arduae difficultates (...) enim oportuit singulari modo confirmari, ne usquam cederet (...). Hinc porro colligamus utilissimam admonitionem de adhibendo scientiae modo. Natura proclives ad curiositatem sumus. Itaque aut omissa aut leviter et neglectim gustata, quae ad aedificationem valet, doctrina, ad quaestiones frivolas abripimur. Accedit deinde audacia ac temeritas, ut de rebus incognitis et occultis non dubitemus statuere. Ex duobus his fontibus nata est nobis bona pars scholasticae theologiae, et quaecumque nugator ille Dionysius de coelestibus hierarchiis comminisci ausus est." (Commentaire des Épitres de Paul, C.O. t. 50, col. 138).

52 E. Forsyth, "Le message prophétique d'A. d'Aubigné", article cité, p. 36. Cela, c'est la position de Bullinger, non celle de Calvin. Voir M. Soulié, op. cit., pp. 138-141.

53 Et c'est Bullinger lui-même qui le rappelle au début de ses Cent Sermons sur l'Apocalypse, tr. fr., J. Crespin (1558).

54 Op. cit., p. 3.

55 "Si on doit garder sobrieté en tous livres de la saincte Ecriture, c'est principalement en ceux esquels Dieu n'a point voulu si manifestement declarer ses secrets, reservant de le faire au temps qu'il a ordonné (...). Mais (...) nous voyons les esprits curieux voltiger en l'air sans avoir rien deferme ni arresté. Parquoy c'est à bon droict que plusieurs ont esté repeus de belles resveries et par ce moyen punis de leur curiosité. Pour ne tomber en de tels dangers, contentons-nous de cognoistre ce que Dieu nous a manifesté de ceste revelation, jusqu'à nostre temps, nous arrestant au but principal pour nous fortifier en foy." Advertissement aux lecteurs touchant la lecture de l'A pocalypse, La Bible qui est toute la saincte Escriture (Genève: H. Estienne, 1565).

56 Cl. G. Dubois, La Conception de l'histoire en France au XVI' siècle (Paris: Nizet, 1977), p. 477.

57 Misères, v. 57.

58 Misères, v. 16.

59 Rappelons, avec Von Rad (op. cit., p. 86), que l'une des formes de la prophétie est le message, à restituer tel quel, ce qui n'empêche pas le prophète d'être libre par ailleurs dans son discours.

60 M.-M. Fragonard (op. cit., p. 72) fait remarquer avec une grande pertinence que le texte des Tragiques se présente comme ayant Dieu pour caution, mais que s'il y a un Dieu au delà du texte, celui-ci représente la seule partie accessible de cet Être.

61 Fers, v. 1446.

62 Feux, v. 26.

63 Op. cit., p. 871, et Augustin, De Genesi ad litteram, IV, 39-50.

64 M. Jeanneret, "Les tableaux spirituels d'A. d'Aubigné”, B.H.R. (1973), pp. 233-245.

65 Voir en particulier "Icones symbolicae. L'image visuelle dans la pensée néo-platonicienne", Symboles de la Renaissance (Paris: Presses de l'École Normale Supérieure, 1976), pp. 1729.

66 E.H. Gombrich, article cité, p. 21.

67 Commentaires du livre de Daniel, C.O., XLI, 37.

68 Article cité, p. 240.

69 Misères, v. 65. 
28 / Renaissance and Reformation

70 Voir F.A. Yates, L'Art de la Mémoire, tr. fr. (Paris: Gallimard, 1975).

71 "Aspetti e orientamenti della poetica protestante francese nel secolo XVI", Studi francesi (1967), pp. 223-245.

72 On ne peut que souscrire à ce jugement de M.-M. Fragonard: "Il (=d'Aubigné) est un exemple frappant du fait que le protestantisme reste marqué d'une sensibilité certaine à des doctrines peu "rationnelles" dont il ne faut pas chercher à cacher le non-conformisme." (op. cit. pp. 839-840). 